PROCEEDINGS OF THE

AMERICAN MATHEMATICAL SOCIETY

Volume 134, Number 8, Pages 2295-2302

S 0002-9939(06)08463-2

Article electronically published on March 20, 2006

\title{
SEMI-CLASSICAL BEHAVIOR OF THE SPECTRAL FUNCTION
}

\author{
IVANA ALEXANDROVA
}

(Communicated by David S. Tartakoff)

\begin{abstract}
We study the semi-classical behavior of the spectral function of the Schrödinger operator with short range potential. We prove that the spectral function is a semi-classical Fourier integral operator quantizing the forward and backward Hamiltonian flow relations of the system. Under a certain geometric condition we explicitly compute the phase in an oscillatory integral representation of the spectral function.
\end{abstract}

\section{INTRODUCTION}

We study the structure of the spectral function associated with the semi-classical Schrödinger operator with short range potential on $\mathbb{R}^{n}$. We prove that the appropriately cut-off spectral function is a semi-classical Fourier integral operator associated to the union of the backward and the forward Hamiltonian flow relations of the principle symbol of the operator. We also show how this allows us, under a certain geometric assumption, to compute the phase in an oscillatory integral representation of the spectral function.

Our result is motivated by the following theorem by Vainberg. In [7, Theorem XII.5] Vainberg considers operators of the form

$$
A=\sum_{i, j=1}^{n} a_{i, j} \frac{\partial^{2}}{\partial x_{i} \partial x_{j}}+\sum_{i=1}^{n} b_{i} \frac{\partial}{\partial x_{i}}+c,
$$

where $a_{i, j}, b_{i}, c \in C^{\infty}\left(\mathbb{R}^{n}\right)$ and $A \equiv-\Delta$ for $\|x\| \geq 1$. He assumes that the energy 1 is non-trapping for the principal symbol of $A$. Vainberg then establishes an asymptotic expansion in $\lambda \rightarrow \infty$ of the spectral function $e_{\lambda}$, which is defined as the Schwartz kernel of $\frac{d E_{\lambda}}{d \lambda}$, where $\left\{E_{\lambda}\right\}$ denotes the spectral family of $A$. Vainberg expresses this asymptotic expansion in the form of a Maslov canonical operator $K_{\Lambda, \lambda}$ associated to a certain Lagrangian submanifold $\Lambda=\Lambda_{y} \subset T^{*} \mathbb{R}^{n}$ and acting on another asymptotic sum in $\lambda$. The Lagrangian submanifold $\Lambda_{y}$ consists of the phase trajectories at energy 1 of the principal symbol of $A$ passing through a fixed base point $x(0)=y$, while the terms of the asymptotic sum on which $K_{\Lambda, \lambda}$ acts solve a recurrent system of transport equations along the phase trajectories of the system.

Received by the editors March 1, 2005

2000 Mathematics Subject Classification. Primary 35P05, 35S99.

Key words and phrases. Semi-classical Schrödinger operators, spectral function, Fourier integral operators. 


\section{THE SEMI-CLASSICAL SPECTRAL FUNCTION}

Here we study the semi-classical behavior of the spectral function of a Schrödinger operator with short range potential at a fixed energy $\lambda>0$. More precisely, we work in the following setting. Let $X$ be a smooth manifold of dimension $n>1$ such that $X$ coincides with $\mathbb{R}^{n}$ on $\mathbb{R}^{n} \backslash B\left(0, R_{0}\right)$ for some $R_{0}>0$, where $B\left(0, R_{0}\right)=\left\{x \in \mathbb{R}^{n}:\|x\|<R_{0}\right\}$ and $\|\cdot\|$ denotes the Euclidean norm on $\mathbb{R}^{n}$. Let $g$ be a Riemannian metric on $X$ which satisfies the condition

$$
g_{i j}(x)=\delta_{i j} \text { for }\|x\|>R_{0} .
$$

Let $V \in C^{\infty}(X ; \mathbb{R})$ be such that for some $\tau>1$ and for every $\alpha \in \mathbb{N}^{n}$

$$
\left|\frac{\partial^{\alpha}}{\partial x^{\alpha}} V(x)\right|_{\mathbb{R}^{n} \backslash B\left(0, R_{0}\right)} \mid \leq C_{\alpha}(1+\|x\|)^{-\tau-|\alpha|}, x \in \mathbb{R}^{n} \backslash B\left(0, R_{0}\right) .
$$

Then the operators $P(h)=\frac{1}{2} h^{2} \Delta_{g}+V, 0<h \leq 1$, admit unique self-adjoint extensions with domains $H_{h}^{2}(X)$. We denote by $\left\{E_{\mu}(h)\right\}$ the spectral family of the operator $P(h)$ and by $e_{\mu}(h)$ the spectral function of $P(h)$, i.e. the Schwartz kernel of $\frac{d E_{\mu}(h)}{d \mu}$. We also let $p(x, \xi)=\frac{1}{2}\|\xi\|_{g}^{2}+V(x)$ be the semi-classical principal symbol of $P$.

We set $R(\lambda \pm i 0, h)=\lim _{\epsilon \downarrow 0}(P(h)-\lambda \mp i \epsilon)^{-1}$, where the limit is taken in the space of bounded operators $\mathcal{B}\left(L_{\alpha}^{2}(X), L_{-\alpha}^{2}(X)\right), \alpha>\frac{1}{2}$, where

$$
L_{ \pm \alpha}^{2}(X)=\left\{f \in L^{2}(X):\left.f\right|_{B\left(0, R_{0}\right)}(1+\|\cdot\|)^{ \pm \alpha} \in L^{2}\left(\mathbb{R}^{n} \backslash B\left(0, R_{0}\right)\right)\right\} .
$$

The operator norm in $\mathcal{B}\left(L_{\alpha}^{2}(X), L_{-\alpha}^{2}(X)\right)$ will be denoted by $\|\cdot\|_{\alpha,-\alpha}$.

Let $H_{p}$ be the Hamiltonian vector field of $p$ and let

$$
\gamma\left(\cdot ; x_{0}, \xi_{0}\right)=\left(x\left(\cdot ; x_{0}, \xi_{0}\right), \xi\left(\cdot ; x_{0}, \xi_{0}\right)\right)
$$

denote the integral curve of $H_{p}$, or (phase) trajectory, with initial conditions $\left(x_{0}, \xi_{0}\right)$ $\in T^{*} X$. We define a non-trapping energy level as follows:

Definition 1. The energy $\lambda>0$ is non-trapping if for every $\left(x_{0}, \xi_{0}\right) \in T^{*} X$, with $\frac{1}{2}\left\|\xi_{0}\right\|_{g}^{2}+V\left(x_{0}\right)=\lambda$ there exists $t_{0}>0$ such that $x\left(s ; x_{0}, \xi_{0}\right) \in \mathbb{R}^{n} \backslash B\left(0, R_{0}\right)$ for every $|s|>t_{0}$. A phase trajectory $\gamma\left(\cdot ; x_{0}, \xi_{0}\right)$ is non-trapped if there exists $t>0$ such that $x\left(s ; x_{0}, \xi_{0}\right) \in \mathbb{R}^{n} \backslash B\left(0, R_{0}\right)$ for all $|s|>t$.

We now choose functions $\chi_{j} \in C_{c}^{\infty}\left(\mathbb{R}^{n} \backslash B\left(0, R_{0}\right) ; \mathbb{R}\right), j=1$, 2, with disjoint supports. We assume that $\lambda>0$ is such that $P-\lambda$ is of principal type. Then it follows that $\Sigma_{\lambda} \stackrel{\text { def }}{=} p^{-1}(\lambda)$ is a $2 n-1$-dimensional submanifold of $T^{*} X$ and

$$
\begin{aligned}
\Lambda_{R}^{+}(\lambda) \stackrel{\text { def }}{=} & \left\{(y,-\eta ; x, \xi):(y, \eta) \in \Sigma_{\lambda},(x, \xi)=\exp \left(t H_{p}\right)(y, \eta), t>0\right\} \\
& \cap T^{*}\left(\operatorname{supp} \chi_{1} \times \operatorname{supp} \chi_{2}\right)
\end{aligned}
$$

and

$$
\begin{aligned}
\Lambda_{R}^{-}(\lambda) \stackrel{\text { def }}{=} & \left\{(x,-\xi ; y, \eta):(x, \xi) \in \Sigma_{\lambda},(y, \eta)=\exp \left(t H_{p}\right)(x, \xi), t<0\right\} \\
& \cap T^{*}\left(\operatorname{supp} \chi_{1} \times \operatorname{supp} \chi_{2}\right)
\end{aligned}
$$

are Lagrangian submanifolds of $T^{*} \mathbb{R}^{n} \times T^{*} \mathbb{R}^{n}$.

To state our main theorem, we further let $\pi_{2}: T^{*} \mathbb{R}^{n} \times T^{*} \mathbb{R}^{n} \rightarrow T^{*} \mathbb{R}^{n}$ denote the canonical projection onto the second factor. We also refer the reader to the Appendix for the definition of the class of semi-classical Fourier integral operators $\mathcal{I}_{h}^{r}, r \in \mathbb{R}$, as well as for a review of the relevant notions from semi-classical analysis. 
We can now state the following

Theorem 1. Let $\rho_{0} \in \Lambda_{R}^{+}(\lambda)$ be such that $\gamma\left(\pi_{2}\left(\rho_{0}\right)\right)$ is non-trapped, and let $\|R(\lambda \pm i 0, h)\|_{\alpha,-\alpha}=\mathcal{O}\left(h^{s}\right), s \in \mathbb{R}$.

Then there exist open sets $W_{ \pm} \in T^{*} \mathbb{R}^{n} \times T^{*} \mathbb{R}^{n}$, such that

$$
\chi_{2} \frac{d E_{\lambda}}{d \lambda} \chi_{1} \in \mathcal{I}_{h}^{1}\left(\mathbb{R}^{2 n},\left(\bar{W}_{+} \cap \Lambda_{R}^{+}(\lambda)\right) \cup\left(\bar{W}_{-} \cap \Lambda_{R}^{-}(\lambda)\right)\right) .
$$

Before proving the theorem, we would like to make two remarks:

Remark 1. The assumption on the polynomial bound of the resolvent is satisfied in a number of interesting situations: at non-trapping energies (see [6, Lemma 2.2]) and at trapping energies $\lambda$ under the assumption that the resonances $\left(\lambda_{j}\right)$ of $P(h)$ are such that $\left|\Im \lambda_{j}\right| \geq C h^{q}$, if $\Re \lambda_{j} \in[\lambda-\epsilon, \lambda+\epsilon]$ for some $\epsilon>0$ (see [5. Proposition $5.1])$. In the latter case, in order to define the resonances by complex scaling, Michel [5] also assumes that there exist $\theta_{0} \in[0, \pi), \epsilon>0$, and $R>0$ such that $V$ extends holomorphically to

$$
D_{\epsilon, R, \theta_{0}}=\left\{r \omega: \omega \in \mathbb{C}^{n}, \operatorname{dist}\left(\omega, \mathbb{S}^{n-1}\right)<\epsilon, r \in \mathbb{C},|r|>R, \arg r \in\left[-\epsilon, \theta_{0}+\epsilon\right)\right\}
$$

and

$$
\exists \beta>0, \exists M>0, \forall x \in D_{\epsilon, R, \theta_{0}},|V(x)| \leq C|x|^{-\beta} .
$$

Remark 2. [1, Theorem 1] roughly says that semi-classical Fourier integral distributions, i.e., Schwartz kernels of the elements of $\mathcal{I}_{h}^{r}\left(\mathbb{R}^{k}, \Lambda\right), r \in \mathbb{R}, k \in \mathbb{N}$, where $\Lambda \subset T^{*} \mathbb{R}^{k}$ is a smooth closed Lagrangian submanifold, can be represented microlocally as oscillatory integrals $\int e^{i \phi(x, \theta) / h} a_{\phi}(x, \theta) d \theta$ with $a_{\phi} \in S(1)$ for every non-degenerate phase function $\phi=\phi(x, \theta)$ which parameterizes $\Lambda$ in the sense that $\Lambda=\left\{\left(x, d_{x} \phi\right): d_{\theta} \phi=0\right\}$ near some point $\rho \in \Lambda$. Furthermore, such a phase function always exists near any point $\rho \in \Lambda$ (see [1. Section 4.1]), and the corresponding symbol $a_{\phi}$ with an asymptotic expansion in $h$ can always be found (see 11, Theorem 1]). Theorem 11 thus implies that the appropriately cut-off spectral function always admits such an oscillatory integral representation. In Lemma 1 below we explicitly compute the phase functions parameterizing certain of these Lagrangian submanifolds.

Proof of Theorem 1, We recall that

$$
\chi_{2} R(\lambda+i 0, h) \chi_{1}-\chi_{2} R(\lambda-i 0, h) \chi_{1}=2 \pi i \chi_{2} \frac{d E_{\lambda}}{d \lambda} \chi_{1}
$$

Since $\gamma\left(\pi_{2}\left(\rho_{0}\right)\right)$ is non-trapped, it follows that there exists an open set $W_{+} \subset$ $T^{*} \mathbb{R}^{n} \times T^{*} \mathbb{R}^{n}$ such that $\gamma\left(\pi_{2}(\rho)\right)$ is non-trapped for every $\rho \in \bar{W}_{+}$. Let $W_{-}=$ $\left\{(x,-\xi ; y, \eta):(y,-\eta ; x, \xi) \in W_{+}\right\}$.

By [2, Lemma 1] and the estimate $\|R(\lambda \pm i 0, h)\|_{\alpha,-\alpha}=\mathcal{O}\left(h^{s}\right)$ we obtain that $K_{\chi_{2} R(\lambda \pm i 0, h) \chi_{1}} \in \mathcal{D}_{h}^{\prime}\left(\mathbb{R}^{2 n}\right)$, where $K_{\chi_{2} R(\lambda \pm i 0, h) \chi_{1}}$ denotes the Schwartz kernel of $\chi_{2} R(\lambda \pm i 0, h) \chi_{1}$. The same proof as in [2, Theorem 2] then shows that

$$
\chi_{2} R(\lambda \pm i 0, h) \chi_{1} \in \mathcal{I}_{h}^{1}\left(\mathbb{R}^{2 n}, \bar{W}_{ \pm} \cap \Lambda_{R}^{ \pm}(\lambda)\right) .
$$

The assumption that $\chi_{1}$ and $\chi_{2}$ have disjoint supports is essential in the proof of [2, Theorem 2]. Since $\Lambda_{R}^{+}(\lambda)$ and $\Lambda_{R}^{-}(\lambda)$ are disjoint, it follows that

$$
\chi_{2} \frac{d E_{\lambda}}{d \lambda} \chi_{1} \in \mathcal{I}_{h}^{1}\left(\mathbb{R}^{2 n},\left(\bar{W}_{+} \cap \Lambda_{R}^{+}(\lambda)\right) \cup\left(\bar{W}_{-} \cap \Lambda_{R}^{-}(\lambda)\right)\right) .
$$


We now turn to showing how the forward and backward flow relations, $\bar{W}_{+} \cap$ $\Lambda_{R}^{+}(\lambda)$ and $\bar{W}_{-} \cap \Lambda_{R}^{-}(\lambda)$, respectively, can be parameterized by non-degenerate phase functions. For that we make the following assumption

Assumption 1. The trajectory $\gamma_{0}\left(\cdot ; y_{0}, \eta_{0}\right)=\left(x_{0}\left(\cdot ; y_{0}, \eta_{0}\right), \xi_{0}\left(\cdot ; y_{0}, \eta_{0}\right)\right) \subset \Sigma_{\lambda}$ with $y_{0} \in \operatorname{supp} \chi_{1}$ and $z_{0} \stackrel{\text { def }}{=} x_{0}\left(t_{0} ; y_{0}, \eta_{0}\right) \in \operatorname{supp} \chi_{2}$ is non-trapped and is contained in a central field, i.e. (see [4, Section 46.C]),

$$
\operatorname{det}\left(\frac{\partial x_{0}}{\partial \eta}\left(t_{0} ; y_{0}, \cdot\right)\right)\left(\eta_{0}\right) \neq 0 \text {. }
$$

By the Implicit Function Theorem, then, there exist open neighborhoods $T \subset \mathbb{R}, Y, Z \subset \mathbb{R}^{n}$, of $t_{0}, y_{0}$, and $z_{0}$, respectively, and a unique function $\eta \in$ $C^{\infty}\left(T \times Y \times Z ; \mathbb{R}^{n}\right)$ such that $\eta\left(t_{0}, y_{0}, z_{0}\right)=\eta_{0}$ and $x(t ; y, \eta(t, y, z))=z,(t, y, z) \in$ $T \times Y \times Z$. We can therefore define the action

$$
S_{\nu}(y, z, t)=\int_{l(t, y, z)} L(\dot{x}, x) d t
$$

over the segment $l(t, y, z)$ from $(y, \eta(t, y, z))$ to $(z, \xi(t ; y, \eta(t, y, z)))$ of the trajectory $\gamma(\cdot ; y, \eta(t, y, z))$ for $(t, y, z) \in T \times Y \times Z$, where $L(\dot{x}, x)=\frac{1}{2}\|\dot{x}\|_{g}^{2}-V(x)+\lambda$ is the Lagrangian associated to the Hamiltonian $p-\lambda$ and $\nu=\operatorname{sgn} t_{0}$.

We now have the following

Lemma 1. Let $\lambda>0$ be such that $P-\lambda$ is of principal type and let $\gamma_{0}, T, Y, Z, \nu$ be as above.

Then $S_{\nu}$ is a non-degenerate phase function and $\Lambda_{S_{\nu}}=\left\{\left(z, y, d_{z} S_{\nu}, d_{y} S_{\nu}\right)\right.$ : $\left.d_{t} S_{\nu}=0,(t, y, z) \in T \times Y \times Z\right\}$ is a closed Lagrangian submanifold such that $\Lambda_{S_{\nu}} \subset \Lambda_{R}^{\nu}(\lambda)$.

Proof. Assumption 1 allows us to apply [4, Theorem 46.C], and we obtain

$$
d_{z} S_{\nu}(t ; y, z)=\xi(t ; y, \eta(t, y, z)) \text {. }
$$

From

$$
\operatorname{det}\left(\frac{\partial x}{\partial \eta}\left(t_{0} ; y_{0}, \cdot\right)\right)\left(\eta_{0}\right) \neq 0
$$

it follows that there exists an open set $U \subset T^{*} \mathbb{R}^{n}$ with $\left(y_{0}, \eta_{0}\right) \in U$ such that

$$
\pi:\left\{\left(y, \eta ; \exp \left(t_{0} H_{p}\right)(y, \eta)\right):(y, \eta) \in U\right\} \rightarrow \mathbb{R}^{n} \times \mathbb{R}^{n}
$$

is a diffeomorphism, where $\pi: T^{*} \mathbb{R}^{n} \times T^{*} \mathbb{R}^{n} \rightarrow \mathbb{R}^{n} \times \mathbb{R}^{n}$ is the canonical projection. Therefore there exists $\psi \in C^{\infty}\left(\mathbb{R}^{2 n}\right)$ such that graph $\exp \left(t_{0} H_{p}\right)=\Lambda_{\psi}$ near $\left(y_{0}, \eta_{0} ; z_{0}, \xi\left(t_{0} ; y_{0}, \eta_{0}\right)\right)$. Since $\exp \left(t_{0} H_{p}\right)$ is a symplectomorphism, it follows that $\operatorname{det}\left(\frac{\partial^{2} \psi(\cdot, \cdot)}{\partial_{x} \partial_{y}}\right)\left(x_{0}, y_{0}\right) \neq 0$. This implies that $\operatorname{det}\left(\frac{\partial \xi}{\partial y}\left(t_{0} ; \cdot \cdot \eta_{0}\right)\right)\left(y_{0}\right) \neq 0$. This, on the other hand, implies that

$$
\operatorname{det}\left(\frac{\partial x}{\partial \eta}\left(-t_{0} ; x_{0}, \cdot\right)\right)\left(\xi_{0}\right) \neq 0, \text { where }\left(x_{0}, \xi_{0}\right)=\exp \left(t_{0} H_{p}\right)\left(y_{0}, \eta_{0}\right) .
$$

Thus we can again apply [4, Theorem 46.C] and obtain

$$
d_{y} S_{\nu}(t ; y, z)=-\eta(t, y, z) .
$$


Lastly, we observe that since $P-\lambda$ is of principal type, it follows that $d d_{t} S_{\nu} \neq$ 0 on $\left\{d_{t} S_{\nu}=p-\lambda=0\right\}$ (see [4, Theorem 46.C]), and therefore $S_{\nu}$ is a nondegenerate phase function. This, together with (11) and (2), implies that, perhaps after decreasing $T \times Y \times Z$ around $\left(t_{0}, y_{0}, z_{0}\right)$,

$$
\begin{aligned}
\Lambda_{S_{\nu}}=\{ & (z, y, \xi(t ; y, \eta(t, y, z)), \eta(t, y, z)): \\
& d_{t} S_{\nu}(t, y, z)=\frac{1}{2}\|\dot{x}(t ; y, \eta(t, y, z))\|_{g}^{2}+V(x(t ; y, \eta(t, y, z)))-\lambda=0, \\
& (t, y, z) \in T \times Y \times Z\} \subset \Lambda_{R}^{\nu}(\lambda),
\end{aligned}
$$

and $\Lambda_{S_{\nu}}$ is a closed Lagrangian submanifold of $T^{*} \mathbb{R}^{2 n}$.

The following lemma describes the microlocal structure of the cut-off spectral function.

Lemma 2. Let $\lambda>0$ be such that $P-\lambda$ is of principal type and $\|R(\lambda+i 0, h)\|_{\alpha,-\alpha}=$ $\mathcal{O}\left(h^{s}\right), s \in \mathbb{R}$. Let $\gamma\left(\cdot ; y_{0}, \eta_{0}\right)$ with $\left(y_{0}, \eta_{0}\right) \in \Sigma_{\lambda}, y_{0} \in \operatorname{supp} \chi_{1}$, and $x\left(t_{0} ; y_{0}, \eta_{0}\right)=$ $z_{0} \in \operatorname{supp} \chi_{2}$ be non-trapped and contained in a central field. Let $\nu=\operatorname{sgn} t_{0}$.

Then there exists $a_{\nu} \in S_{2 n+1}^{\frac{n+3}{2}}(1) \cap C_{c}^{\infty}\left(\mathbb{R}^{2 n+1}\right)$ such that

$$
\left(\chi_{2} \otimes \chi_{1}\right) e_{\lambda}=\int e^{\frac{i}{h} S_{\nu}(y, x, t)} a_{\nu}(y, x, t) d t
$$

microlocally near $\left(y_{0},-\eta_{0} ; z_{0}, \xi\left(t_{0} ; y_{0}, \eta_{0}\right)\right)$ if $t_{0}>0$ or near $\left(z_{0},-\xi\left(t_{0} ; y_{0}, \eta_{0}\right) ; y_{0}, \eta_{0}\right)$ if $t_{0}<0$.

Proof. With Theorem 1 and Lemma 1 the conditions of [1, Theorem 1] are satisfied, and we obtain from [1, Theorem 1] that there exist $a_{\nu} \in S_{2 n+1}^{\frac{n+3}{2}}(1) \cap C_{c}^{\infty}\left(\mathbb{R}^{2 n+1}\right)$ such that

$$
\left(\chi_{2} \otimes \chi_{1}\right) e_{\lambda}=\int e^{\frac{i}{h} S_{\nu}(y, x, t)} a_{\nu}(y, x, t) d t
$$

microlocally near $\left(y_{0},-\eta_{0} ; z_{0}, \xi\left(t_{0} ; y_{0}, \eta_{0}\right)\right)$ for $t_{0}>0$ and near $\left(z_{0},-\xi\left(t_{0} ; y_{0}, \eta_{0}\right)\right.$; $\left.y_{0}, \eta_{0}\right)$ for $t_{0}<0$.

\section{Appendix A. Elements of Semi-Classical analysis}

In this section we recall some of the elements of semi-classical analysis which we have used here. First we define two classes of symbols

$$
\begin{aligned}
S_{2 n}^{m}(1)=\left\{a \in C^{\infty}\left(\mathbb{R}^{2 n} \times\left(0, h_{0}\right]\right): \forall \alpha, \beta \in \mathbb{N}^{n},\right. \\
\left.\sup _{(x, \xi, h) \in \mathbb{R}^{2 n} \times\left(0, h_{0}\right]} h^{m}\left|\partial_{x}^{\alpha} \partial_{\xi}^{\beta} a(x, \xi ; h)\right| \leq C_{\alpha, \beta}\right\}
\end{aligned}
$$

and

$$
\begin{array}{r}
S^{m, k}\left(T^{*} \mathbb{R}^{n}\right)=\left\{a \in C^{\infty}\left(T^{*} \mathbb{R}^{n} \times\left(0, h_{0}\right]\right): \forall K \Subset \mathbb{R}^{n}, \forall \alpha, \beta \in \mathbb{N}^{n},\right. \\
\left.\sup _{x \in K}\left|\partial_{x}^{\alpha} \partial_{\xi}^{\beta} a(x, \xi ; h)\right| \leq C_{\alpha, \beta} h^{-m}\langle\xi\rangle^{k-|\beta|}\right\},
\end{array}
$$


where $h_{0} \in(0,1]$ and $m, k \in \mathbb{R}$. For $a \in S_{2 n}^{m}(1)$ or $a \in S^{m, k}\left(T^{*} \mathbb{R}^{n}\right)$ we define the corresponding semi-classical pseudodifferential operator of class $\Psi_{h}^{m}\left(1, \mathbb{R}^{n}\right)$ or $\Psi_{h}^{m, k}\left(\mathbb{R}^{n}\right)$, respectively, by setting

$$
O p_{h}(a) u(x)=\frac{1}{(2 \pi h)^{n}} \iint e^{\frac{i\langle x-y, \xi\rangle}{h}} a(x, \xi ; h) u(y) d y d \xi, u \in \mathcal{S}\left(\mathbb{R}^{n}\right),
$$

and extending the definition to $\mathcal{S}^{\prime}\left(\mathbb{R}^{n}\right)$ by duality. Below we shall work only with symbols which admit asymptotic expansions in $h$ and with pseudodifferential operators which are quantizations of such symbols. For $A \in \Psi_{h}^{m}\left(1, \mathbb{R}^{n}\right)$ or $A \in \Psi_{h}^{m, k}\left(\mathbb{R}^{n}\right)$, we shall use $\sigma_{0}(A)$ and $\sigma(A)$ to denote its principal symbol and its complete symbol, respectively. A semi-classical pseudodifferential operator will be called of principal type if its principal symbol $a_{0}$ satisfies

$$
a_{0}=0 \Longrightarrow d a_{0} \neq 0 \text {. }
$$

For $a \in S^{m, k}\left(T * \mathbb{R}^{n}\right)$ or $a \in S_{2 n}^{m}(1)$ we define:

$$
\begin{aligned}
& \operatorname{ess-supp}_{h} a \\
& =\left\{(x, \xi) \in T^{*} \mathbb{R}^{n} \mid \exists \epsilon>0 \partial_{x}^{\alpha} \partial_{\xi}^{\beta} a\left(x^{\prime}, \xi^{\prime}\right)=\mathcal{O}_{C(B((x, \xi), \epsilon))}\left(h^{\infty}\right), \forall \alpha, \beta \in \mathbb{N}^{n}\right\}^{c} \\
& \cup\left(\left\{(x, \xi) \in T^{*} \mathbb{R}^{n} \backslash\{0\} \mid \exists \epsilon>0 \partial_{x}^{\alpha} \partial_{\xi}^{\beta} a\left(x^{\prime}, \xi^{\prime}\right)=\mathcal{O}\left(h^{\infty}\langle\xi\rangle^{-\infty}\right),\right.\right. \\
& \text { uniformly in } \left.\left.\left(x^{\prime}, \xi^{\prime}\right) \text { such that }\left\|x-x^{\prime}\right\|+\frac{1}{\left\|\xi^{\prime}\right\|}+\left|\frac{\xi}{\|\xi\|}-\frac{\xi^{\prime}}{\left\|\xi^{\prime}\right\|}\right|<\epsilon\right\} / \mathbb{R}_{+}\right)^{c} \\
& \subset T^{*} \mathbb{R}^{n} \sqcup S^{*} \mathbb{R}^{n},
\end{aligned}
$$

where we define $S^{*} \mathbb{R}^{n}=\left(T^{*} \mathbb{R}^{n} \backslash\{0\} / \mathbb{R}_{+}\right.$and denote by $\bullet$ c the complement of the set - For $A \in \Psi_{h}^{m, k}\left(\mathbb{R}^{n}\right)$ or $A \in \Psi_{h}^{m}\left(1, \mathbb{R}^{n}\right)$ we then define its semi-classical wavefront set

$$
W F_{h}(A)=\operatorname{ess}^{-\operatorname{supp}_{h}} a, A=O p_{h}(a)
$$

We also define the class of semi-classical distributions $\mathcal{D}_{h}^{\prime}\left(\mathbb{R}^{n}\right)$ with which we will work here

$$
\begin{gathered}
\mathcal{D}_{h}^{\prime}\left(\mathbb{R}^{n}\right)=\left\{u \in C_{h}^{\infty}\left((0,1] ; \mathcal{D}^{\prime}\left(\mathbb{R}^{n}\right)\right): \forall \chi \in C_{c}^{\infty}\left(\mathbb{R}^{n}\right) \exists N \in \mathbb{N} \text { and } C_{N}>0:\right. \\
\left.\left|\mathcal{F}_{h}(\chi u)(\xi)\right| \leq C_{N} h^{-N}\langle\xi\rangle^{N}\right\},
\end{gathered}
$$

where

$$
\mathcal{F}_{h}(\chi u)(\xi)=\left\langle e^{-\frac{i}{h}\langle\cdot, \xi\rangle}, \chi u\right\rangle
$$

and $\langle\cdot, \cdot\rangle$ denotes the distribution pairing, with the obvious extension of this definition to $\mathcal{E}_{h}^{\prime}\left(\mathbb{R}^{n}\right)$. Everywhere here we work with the $L^{2}$-based semi-classical Sobolev spaces $H_{h}^{s}\left(\mathbb{R}^{n}\right), s \in \mathbb{R}$, which consist of the distributions $u \in \mathcal{E}_{h}^{\prime}\left(\mathbb{R}^{n}\right)$ such that

$$
\|u\|_{H_{h}^{s}\left(\mathbb{R}^{n}\right)}^{2} \stackrel{\text { def }}{=} \frac{1}{(2 \pi h)^{n}} \int_{\mathbb{R}^{n}}\left(1+\|\xi\|^{2}\right)^{s}\left|\mathcal{F}_{h}(u)(\xi)\right|^{2} d \xi<\infty .
$$

We shall say that $u=v$ microlocally (or $u \equiv v$ ) near an open or closed set $U \subset T^{*} \mathbb{R}^{n}$, if $P(u-v)=\mathcal{O}\left(h^{\infty}\right)$ in $C_{c}^{\infty}\left(\mathbb{R}^{n}\right)$ for every $P \in \Psi_{h}^{0}\left(1, \mathbb{R}^{n}\right)$ such that

$$
W F_{h}(P) \subset \tilde{U}, \bar{U} \Subset \tilde{U} \Subset T^{*} \mathbb{R}^{n}, \tilde{U} \text { open. }
$$


We shall also say that $u$ satisfies a property $\mathcal{P}$ microlocally near an open set $U \subset$ $T^{*} \mathbb{R}^{n}$ if there exists $v \in \mathcal{D}_{h}^{\prime}\left(\mathbb{R}^{n}\right)$ such that $u=v$ microlocally near $U$ and $v$ satisfies property $\mathcal{P}$.

We now define global semi-classical Fourier integral operators.

Definition 2. Let $\Lambda \subset T^{*} \mathbb{R}^{k}$ be a smooth closed Lagrangian submanifold with respect to the canonical symplectic structure on $T^{*} \mathbb{R}^{k}$. Let $r \in \mathbb{R}$. Then the space $I_{h}^{r}\left(\mathbb{R}^{k}, \Lambda\right)$ of semi-classical Fourier integral distributions of order $r$ associated to $\Lambda$ is defined as the set of all $u \in \mathcal{E}_{h}^{\prime}\left(\mathbb{R}^{k}\right)$ such that

$$
\left(\prod_{j=0}^{N} A_{j}\right)(u)=\mathcal{O}_{L^{2}\left(\mathbb{R}^{k}\right)}\left(h^{N-r-\frac{k}{4}}\right), h \rightarrow 0,
$$

for all $N \in \mathbb{N}_{0}$ and for all $A_{j} \in \Psi_{h}^{0}\left(1, \mathbb{R}^{k}\right), j=0, \ldots, N-1$, with compact semi-classical wavefront sets and principal symbols vanishing on $\Lambda$, and any $A_{N} \in$ $\Psi_{h}^{0}\left(1, \mathbb{R}^{k}\right)$ with a compact semi-classical wavefront set.

A continuous linear operator $C_{c}^{\infty}\left(\mathbb{R}^{m}\right) \rightarrow \mathcal{D}_{h}^{\prime}\left(\mathbb{R}^{l}\right), m, l \in \mathbb{N}$, whose Schwartz kernel is an element of $I_{h}^{r}\left(\mathbb{R}^{m+l}, \Lambda\right)$ for some Lagrangian submanifold $\Lambda \subset T^{*} \mathbb{R}^{m+l}$ and some $r \in \mathbb{R}$, will be called a global semi-classical Fourier integral operator of order $r$ associated to $\Lambda$. We denote the space of these operators by $\mathcal{I}_{h}^{r}\left(\mathbb{R}^{m+l}, \Lambda\right)$.

We extend these notions to compact manifolds through the following definition of semi-classical pseudodifferential operators on compact manifolds. Let $M$ be a smooth compact manifold and $\kappa_{j}: M_{j} \rightarrow X_{j}, j=1, \cdots, N$, a set of local charts. A linear continuous operator $A: C^{\infty}(M) \rightarrow \mathcal{D}_{h}^{\prime}(M)$ belongs to $\Psi_{h}^{m}(1, M)$ or $\Psi_{h}^{m, k}\left(T^{*} M\right)$ if for all $j \in\{1, \cdots, N\}$ and $u \in C_{c}^{\infty}\left(M_{j}\right)$ we have $A u \circ \kappa_{j}^{-1}=$ $A_{j}\left(u \circ \kappa_{j}^{-1}\right)$ with $A_{j} \in \Psi_{h}^{m}\left(1, X_{j}\right)$ or $A_{j} \in \Psi_{h}^{m, k}\left(X_{j}\right)$, respectively, and $\chi_{1} A \chi_{2}$ : $\mathcal{D}_{h}^{\prime}(M) \rightarrow h^{\infty} C^{\infty}(M)$ if supp $\chi_{1} \cap \operatorname{supp} \chi_{2}=\emptyset$.

Lastly, we define the microlocal equivalence of two semi-classical Fourier integral operators.

Definition 3. Let $M_{j}, j=1,2$, be smooth manifolds, $\Lambda \subset T^{*} M_{1} \times T^{*} M_{2}$ a Lagrangian submanifold, and $T, T^{\prime} \in \mathcal{I}_{h}^{r}\left(M_{1} \times M_{2}, \Lambda\right)$ for some $r \in \mathbb{R}$. For open or closed sets $U \subset T^{*} M_{1}$ and $V \subset T^{*} M_{2}$, the operators $T$ and $T^{\prime}$ are said to be microlocally equivalent near $U \times V$ if there exist open sets $\tilde{U} \Subset T^{*} M_{1}$ and $\tilde{V} \Subset T^{*} M_{2}$ with $\bar{U} \Subset \tilde{U}$ and $\bar{V} \Subset \tilde{V}$ such that for any $A \in \Psi_{h}^{0}\left(1, M_{1}\right)$ and $B \in \Psi_{h}^{0}\left(1, M_{2}\right)$ with $W F_{h}(A) \subset \tilde{U}$ and $W F_{h}(B) \subset \tilde{V}$, we have that

$$
B\left(T-T^{\prime}\right) A=\mathcal{O}\left(h^{\infty}\right): \mathcal{D}_{h}^{\prime}\left(M_{1}\right) \rightarrow C^{\infty}\left(M_{2}\right) .
$$

We shall also write $T \equiv T^{\prime}$ near $V \times U$.

\section{REFERENCES}

[1] Alexandrova, Ivana. Semi-Classical Wavefront Set and Fourier Integral Operators. To appear in Canadian Journal of Mathematics.

[2] Alexandrova, Ivana. Structure of the Semi-Classical Amplitude for General Scattering Relations. Communications in Partial Differential Equations. 2005, 30 (10-12), 1505-1535. MR 2182302

[3] Alexandrova, Ivana. Structure of the Short Range Amplitude for General Scattering Relations. Preprint mathAP/0411599 on arxiv.org.

[4] Arnold, Vladimir. Mathematical Methods of Classical Mechanics. Springer-Verlag. New York, 1980. MR0690288 (57:14033b) 
[5] Michel, Laurent. Semi-classical Behavior of the Scattering Amplitude for Trapping Perturbations at Fixed Energy. Canadian Journal of Mathematics. 2004, 56, (4), 794-824. MR 2074047 (2005e:35176)

[6] Robert, Didier and Tamura, Hideo. Asymptotic Behavior of Scattering Amplitudes in SemiClassical and Low Energy Limits. Annales de l'Institut Fourier. 1989, 39, (1), 155-192. MR:1011982 (91c:35116)

[7] Vainberg, Boris. Asymptotic Methods in Equations of Mathematical Physics. Gordon and Breach Science Publishers. New York. 1989. MR.1054376 (91h:35081)

Department of Mathematics, University of Toronto, Toronto, Ontario, Canada M5S 3G3

E-mail address: alexandr@math.toronto.edu 